\title{
Car parking is a clinical quality issue
}

In the latest of a monthly series in which patients and carers set learning points for readers, David Gilbert urges healthcare staff to think again about their role in improving clinical outcomes. For more information about the series, contact Rosamund Snow, patient editor, rsnow@bmj.com.

\section{David Gilbert}

I know of an NHS organisation with a large team overseeing clinical quality. In an office nearby, a medium sized team focuses on improving the "patient experience." A small team over the road oversees "patient and public involvement." It reminds me of Goldilocks and the three bears.

More seriously, it reminds me of my mother.

During her final 10 years, she had severe depression, deafness, and a form of Parkinson's disease that left her body rigid; she was increasingly unable to swallow. She saw one doctor for this, another for that, and another for something else. Some of them seemed as frustrated as we were, as we tried to find our way to get what we needed amid a multitude of nurses, physiotherapists, speech and language therapists, and administration staff. We were certainly "put at the centre" of our care-but it was like being surrounded rather than in a partnership.

As she moved from warden assisted housing, to a residential home, and then a nursing home, it became even more complicated, because as a family as we had to build and re-build relationships. The disconnection between different parts of the system-actually, different systems-was dispiriting.

The various doctors we saw (GP, neurologist, psychiatrist) often seemed unaware of who else we had seen. My mum and her notes were hardly ever in the same room at the same time.

And then there was the car parking.

One day, we had an early neurology outpatients appointment that I couldn't shift to a better time ("that's all we have, take it or leave it" said the secretary). I had to get a nurse at the care home to wake her early because she took two and a half hours to dress, bathe, and eat. She hadn't slept owing to anxiety about the appointment. I took a day off work, picked her up early, and spent 90 minutes circling the car park for a space. She grew more nervous. She didn't want me to drop her off and nobody seemed able to help.

When we made it to outpatients, I was relieved by the irony that the consultant was late. We arrived just in time to hear mum's name being called. She was in tears and clung onto my arm as she limped in. The doctor didn't ask why she seemed particularly flustered, perhaps logging it as part of her symptomology without understanding the context.
Mum was too upset to comprehend, or answer, the doctor's questions and mumbled responses. I fear she got the wrong prescription because the doctor did not get the full picture. She remembered nothing of what was said. She was so upset that she arranged an unscheduled, and what should have been entirely unnecessary, emergency appointment with her psychiatrist. For us, this meant another journey, which included more time off work for me and a repeat of the car parking fiasco. This seemed a total waste of hospital resources.

There are many things to take from this- the needlessness of some outpatient appointments, the inflexibility of appointments, and the waste of resources - all of which indicate a system built around institutional rather than patient need. And what about the car parking?

Car parking is a clinical quality issue. This "access issue" affected our "experience" and led to poor "involvement" (mum literally being unable to find her voice or listen), poor quality care, and poor outcomes. My mum was given drugs she did not know how to take, information she was unable to follow, and advice that she was ill equipped to deal with. Her physical and psychological health that day were directly attributable to the car parking. The stress of dealing with the system was too much for her. This was one of many "experiences."

I am left wondering what doctors think about access problems that have a knock-on effect on care. Is there a professional blind spot? Are systems problems ignored because doctors, like patients, feel powerless to influence them?

Returning to those separate departmental teams—dealing with clinical quality, improving patients' experiences, and promoting patient and public involvement. It seems that the way the system tackles improvement mirrors the fragmentation of service delivery. If my mum had known that we "need" three different teams to help improve care, each tackling a different bit of what happened that day, she would have cried even more.

Competing interests: I have read and understood BMJ Group policy on declaration of interests and declare the following interests: none.

Cite this as: BMJ 2015:350:h1312

๑ BMJ Publishing Group Ltd 2015 


\section{The bottom line}

- The role of doctors and clinical staff: could you find ways to acknowledge, or factor in, the stresses and strains of making it to appointments? Thirty seconds asking us how the journey had been would have put us at ease and provided useful context for the consultation

- The role of non-clinical staff: administration staff, receptionists, and car park attendants could make things easier with a quick "I know it must have been tricky finding a space today." A smile at the reception desk would have gone a long way. Can you as a doctor support other staff to feel more valued? Happy workers make happier patients

- Simple solutions: are there simple solutions, such as providing better information about parking or park-and-ride facilities? How about volunteers accompanying people from the car park to the outpatient department (plus a glass of water for my mum, please)? Often patients can help with solutions if asked

- Improving the system: although individual doctors might be doing a good job, how could they help join up the system? How might neurologists, psychiatrists, and GPs communicate better with each other? Could car parking (and transport) be more of a corporate priority? That can only happen if all stakeholders-local authority, commissioners, parking authorities, finance directorates, clinicians, non-clinical staff, and patients and carers-are at the table

- Improving engagement: do you know about the various teams in your organisation trying to improve quality or involve patients and the public? How might their efforts be made more useful to you? Can you help find better ways for people like my mum to have their views listened to, and more importantly, acted on? We received a postal questionnaire and mum rated the hospital highly, largely because she liked the doctor and didn't want to make a fuss. Her experience told a different story. Ideas that could have helped were lost 